\title{
Application Analysis of Big Data in Traffic Planning Industry
}

\author{
Bo Gao' \\ ${ }^{1}$ Liaoning Police College, Dalian Liaoning, 116036
}

Keywords: application analysis; big data; traffic planning industry

\begin{abstract}
In the era of big data, data resources play an increasingly important role in the national economic and social development, especially in the transportation industry. Data has a very important significance in the field of intelligent transportation. The application of big data in intelligent transportation will have a tremendous impact on traffic patterns and concepts, and it can better solve or alleviate many problems facing traffic. Based on big data applications, the development of intelligent transportation systems was studied.
\end{abstract}

\section{Introduction}

At present, the transportation industry has accumulated a great deal of transportation infrastructure data, as well as dynamic data collection of traffic flow, people flow and logistics. The storage format is mainly in the form of paper documents. It is inconvenient to perform maintenance and management. With the huge amount of data, the later work will be more and more difficult to carry out. With such a large amount of data, there is no systematic management and it is difficult to support planning and research work in the transportation industry. At the same time, due to various reasons such as the system, there are still some deficiencies such as unreasonable transport structure, separate management of different modes of transport, overlapping of similar transport modes, and unclear functions, which limits the sustainable development of the industry in the future. In order to accelerate the transformation and upgrading, strengthen the optimization functions, and adapt to the development trend, the transportation industry must rely on the background and advantages of big data to construct a planning information platform with an integrated transportation system [1].

\section{The Concept of Big Data}

Big data mainly refers to the huge amount of data, which cannot pass through mainstream software, and it is difficult to exert the value and role of this data. Through its reasonable processing and sorting, it is in the management and decision-making of the enterprise. Big data is the integration of data sets. Because of its largeness, it is difficult to achieve its processing through the current database management tools. On the one hand, it reflects the difficulty of its management, and on the other hand, it also shows its use value to some extent. The characteristics of big data are mainly reflected in four aspects: 1 The volume of data is huge; 2 There are many types of data, including video, location, pictures, network logs and other aspects of information; 3 poor value-intensive, have a higher Business value, such as video data, is generated in the continuous monitoring process, but the valuable part may only be a second or second; 4 has a very fast processing speed and has a big difference compared with traditional data mining technology. .

Open data mainly refers to the relevant organizations to achieve the opening of various types of raw data information, generally through the form of portal sites to achieve, thereby enhancing the readability of the data. In addition, in the portal site, there is corresponding data extraction and mining tools, users or businesses can accord to their own needs, through the Internet-related agreements, rules to complete the addition, storage and development of data. In addition, the government also needs to show the principle of data opening, protect intellectual property rights, formulate relevant reward systems for improving data sharing, and improve the integration and openness of data information [2].

Data mining is mainly to find valuable and understandable non-trivial knowledge from huge data 
information. With a variety of different analysis methods and tools, the relationship between data and models is established from massive data sources. Data mining belongs to a new type of technology in recent years. With data mining, people can apply more knowledge research results in data processing and provide sufficient data support for scientific decision-making.

\section{The Acquisition Characteristics of Traffic Big Data}

Acquisition of traffic big data from the point of view of access to big data, there is a big difference from traditional sampling survey data. In the past, traffic surveys were often concentrated on major indicators such as traffic patterns and traffic volumes. Traffic Big Data opens up a wide range of sample acquisition channels and builds traffic models through multidimensional traffic data. Because the information obtained by big data has the characteristics of "mass" and "diversity", its sampling range is infinitely close to the whole sample, and its accuracy has been greatly improved compared to the traditional sample survey. In general, traffic big data can be divided into the following types: 1 demand data generated by mobile terminals, demand data provided by mobile terminal software such as inquiries, bookings, and calling vehicles; 2 on-board travel data, mainly various types of vehicle-mounted vehicles Internet, satellite positioning system and other data; 3 traffic supervision data, such as license plate recognition, video camera monitoring, access control traffic, and sense coils; 4 traffic consumption data, such as parking, bus cards, taxi mileage and station charges Data; 5 Traffic violations and accident data, including the specific time, location, type, and impact on traffic and traffic accidents; 6 Traditional survey data, such as regional master planning, socio-economic survey data, etc.

From the characteristics of traffic big data, big data has the following main features:

The big data survey is different from the traditional traffic data survey. The data acquisition is mainly achieved through long-term objective analysis of the Internet, terminal software, and other long-term objective. The traditional traffic data survey is often the subjective response of the surveyee in the short term, which improves the data [3].

From the above analysis, big data is acquired through multi-dimensional channels. It is a comprehensive response of the new channels such as Internet+ and the Internet of Things to urban transport, and the traffic data information obtained from a multidimensional perspective is analyzed through statistical analysis, simulation regression, and other means. Embody the multi-dimensional characteristics of traffic, and data can also be mutually authenticated.

Big data through a variety of Internet +, Internet of Things and other means of full expression of urban transport, eliminating the sample extraction, survey visits, fill in the form of input and other survey work, while avoiding the traditional traffic survey sample limited, improve traffic data Global collection of information.

Big data can monitor and manage urban traffic in real time, which is helpful for planners to grasp the overall development trend of the survey target, so as to verify each other's data and the interference caused by low-probability data.

\section{The Application of Big Data in Traffic}

The application of big data technology in traffic is mainly reflected in the following aspects:

Big data has the characteristics of virtualization and can eliminate regional restrictions. In order to facilitate management, the state usually divides the administrative regions. However, in this division model, local governments are more to maximize the interests within the jurisdiction, and lack attention to the construction of basic transport facilities in the border areas and traffic routes. . Because of the virtual nature of big data, cross-regional information management can be realized, and multiple parties can abide by the principle of information sharing, making cross-regional administrative management issues effectively resolved.

Big data has the advantages of combination efficiency and information integration. Currently, the main points of traffic management in China are in different management departments, and each department has an independent information management system. In the storage of data information, 
more Concentration on vertical services leads to poor linkage of information between systems. Through big data, traffic information systems can be built toward comprehensiveness and three-dimensionality, and the integration of data and information in different regions and different areas can be realized, and the overall function of traffic can be brought into play, bringing more development opportunities.

The intelligence of traffic big data can significantly improve the allocation of transportation resources. The previous transportation management was mainly achieved through the human approach, resulting in a lack of dynamics in traffic management. With the analysis of big data, we can formulate an ideal coordination and overall plan for traffic management, reduce the consumption of human and material resources in the transportation process, and improve the rationality of transportation resources [4].

Through the predictability of big data, the level of traffic prediction can be significantly improved. In the past, in terms of improving the traffic environment, it was mainly achieved by increasing the intensity of infrastructure construction, such as widening the road. However, this method has many limitations. In addition, the actual planning scheme may not necessarily meet the needs of the future. With the help of big data to analyze the data, the corresponding traffic prediction model can be established to simulate the future traffic conditions and then verify the feasibility of the scheme.

\section{The Impact of Big Data on the Revolution of Urban Transport Theory}

The application of big data in the field of transportation has greatly changed the limitations of traditional urban transport planning theory research. On the one hand, big data makes urban transport planning methods more objective, true, accurate, and in line with the development of the times; on the other hand, Big Data breaks through the research perspective of traditional urban transport planning, and makes the development of urban transport planning theory full of infinite possibilities. From the current situation, the impact of big data on the reform of urban transport planning theory is mainly reflected in the following aspects.

In traditional transportation planning theory, sampling survey is the basic way to obtain data, and it is also the most convenient way for researchers to operate. However, the sampling survey itself has certain limitations and consumes a lot of manpower and material resources. What's more, the complexities of today's urban transportation planning can no longer be the same, and the investigation work will be different for different regions, levels, and stages. To achieve convergence, and the survey results will have a certain degree of subjectivity, can not objectively show the real data results, which makes the city traffic planning data, there are various types of contradictions, affecting the overall regional transport planning. As a result, big data came into being, and its application completely made up for the shortcomings of traditional sample surveys. In a large number of areas, especially in areas with higher levels of specialization, big data has been able to replace traditional sampling and become the new darling of the times. Since big data can cover almost all personal traffic information within the scope of research, it can control the characteristics of urban residents' travel as a whole, which is conducive to doing top-level design and planning the future development direction and trend of urban transport. It can also be meticulous, taking into consideration the different groups of people and giving them the best experience [5].

Traditional sampling surveys have considerable limitations. First, consume labor. The effective implementation of the investigation work requires a large number of staff, labor costs are high; secondly, it takes time. The investigation work takes a very long time. The investigation takes a long time, the interval is long, and the processing time is long. It cannot effectively ensure the real-time performance. Finally, it is error. The error rate existing in the traditional sampling survey will greatly affect the accuracy of urban transport planning. With the influx of the Internet + , this society has long been in an ever-changing state. With these lagging data, researchers cannot precisely regulate the traffic ecology of the entire city. This will make the planning efficiency of urban transportation always low, unable to meet the needs of modern urban transport planning.

As we all know, the complexity of the current urban traffic situation is increasing day by day. 
Not only do we need to consider the balance of supply and demand for residents' transportation, but they also involve all aspects of society. This will give researchers some trouble in planning urban transportation. Big data can greatly ease the pressure of urban transport research, enabling researchers to devote more time to specialized research and improve research efficiency. This makes the breakthrough of urban traffic complexity theory and the change of urban traffic planning theory become possible.

Researchers must obtain the most complete and practical plan for urban transport planning, and be able to apply it to the real traffic environment. The following points need to be fulfilled: 1 to predict in time. In the study of urban transport planning, all possible adverse factors should be pre-judged in advance, and the treatment plan can be given according to the actual situation, so as to prevent problems before they occur. This requires us to monitor and measure urban traffic all-weather, so as to ensure the overall coordination of urban traffic conditions, rather than relying only on regular sample surveys; 2 Add elements. A detailed, complete, and idealized urban transportation system can not only consider the supply and demand of urban transportation, but also take into account all the important factors related to it, such as environmental impact, passenger evaluation, and construction of transportation service websites. Use big data thinking and comprehensive analysis to make the best possible solution.

\section{Conclusion}

Big data can further accelerate the development of urban transport planning theory, and is expected to break through the complexity theory, and finally achieve a new revolution in urban transport planning theory. This is not only the need for the development of urban transport ecology, but also the inevitable result of technological development and theoretical progress. With the promotion of the consciousness of change and the cooperation of managers, engineering technicians and researchers, the new future of urban transport planning theory will eventually become a reality.

\section{References}

[1] Ji Zhendong. Application of Big Data Analysis Cloud Platform Technology in Intelligent Transportation [J]. Silicon Valley, 2015(01).99

[2] Cao Xingyan. Application of Big Data Processing Platform Based on Transportation Industry [J]. Railway Communication Signal Engineering Technology, 2016(02).123

[3] Li Jianguo. Application and development of big data in intelligent transportation [J].Science \& Technology, 2015(07).67

[4] Zhou Hao. Application of Big Data in Public Transportation [J]. Smart City, 2016 (07). 58

[5] Liu Zongxi. Cold thinking on "big data" in traffic planning - Application of Shanghai traffic planning For example [J]. Shanghai Urban Management, 2017, 26(3):62-66. 